\title{
Editor's Note: Special Section on Data-Flow for Multicore
}

The International Journal of Parallel Programming journal gratefully acknowledges the editorial work of the following scholars on this special section dedicated to DataFlow for Multicore:

Costas N Kyriacou, Frederick University, Cyprus

Paraskevas Evripidou, University of Cyprus, Cyprus

Samer Arandi, An-Najah National University, Palestine

The 4 papers in this section include:

- Architectural Support for Fault Tolerance in a Teradevice Dataflow System by Sebastian Weis, Arne Garbade, Bernhard Fechner, Avi Mendelson, Roberto Giorgi, Theo Ungerer

- SCnC: Efficient Unification of Streaming with Dynamic Task Parallelism by Dragoş Sbîrlea, Jun Shirako, Ryan Newton, Vivek Sarkar

- Integrating Transactions into the Data-Driven Multi-threading Model Using the TFlux Platform by Andreas Diavastos, Pedro Trancoso, Mikel Luján, Ian Watson

- The Design and Implementation of TIDeFlow: A Dataflow-Inspired Execution Model for Parallel Loops and Task Pipelining by Daniel Orozco, Elkin Garcia, Robert Pavel, Jaime Arteaga, Guang Gao 